\title{
A automedicação infantil ocasionada pelos pais no Brasil
}

\author{
Child self-medication caused by parents in Brazil
}

La automedicación infantil provocada por los padres en Brasil

Recebido: 17/11/2021 | Revisado: 25/11/2021 | Aceito: 26/11/2021 | Publicado: 29/11/2021

Jaqueline Carlan Marques Alves

ORCID: https://orcid.org/0000-0002-0998-9105

Universidade Nilton Lins, Brasil

E-mail: j.carlan07@gmail.com

Edivane Queiroz Magalhães

ORCID: https://orcid.org/0000-0002-9866-0845

Universidade Nilton Lins, Brasil

E-mail: edivaneqm_@hotmail.com

Omero Martins Rodrigues Júnior

ORCID: https://orcid.org/0000-0002-8552-3278

Universidade Nilton Lins, Brasil

E-mail: omeromartins.farma@gmail.com

\begin{abstract}
Resumo
Automedicar-se é o ato de ingerir remédios para aliviar sintomas, sem qualquer orientação médica no diagnóstico, prescrição ou acompanhamento do tratamento. Portanto o objetivo desse trabalho é identificar os fatores associados à prática da automedicação ocasionada pelos pais, os fármacos mais utilizados, os motivos e relatar a importância do uso racional de medicamentos. A metodologia empregada é de uma revisão bibliográfica nos bancos de dados como: a disponibilidade de informações médicas na internet cria um ambiente propício para a pessoa fazer diagnóstico e se medicar por conta própria. Esses fatores tornaram o uso indiscriminado de medicamentos um dos principais problemas da saúde no Brasil. Todo remédio possui efeitos colaterais e, quando ingerido de forma incorreta, pode causar mais malefícios que benefícios ao organismo. Dentre os riscos da automedicação, podemos citar as intoxicações e a dificuldade de diagnosticar certas doenças devido ao fato de que alguns medicamentos podem mascarar sintomas importantes. Atualmente muitos dos responsáveis pelas crianças empregam a automedicação para alívio dos sintomas como cefaleia, resfriado e dor, recorrendo a farmácias domiciliares, as quais contêm analgésicos, antigripais e anti-inflamatórios que podem representar risco para as crianças. No Brasil, como na maioria dos países, os medicamentos são o principal agente de intoxicação. Eles correspondem a 27,86\% dos casos registrados por ano, segundo dados do Sistema Nacional de Informações Toxico Farmacológicas (Sinitox). Do total de casos por intoxicação de medicamentos, crianças menores de cinco anos representam $35 \%$.
\end{abstract}

Palavras-chave: Automedicação; Uso inadequado de medicamentos sob prescrição; Medicamentos sem prescrição.

\begin{abstract}
Self-medicating is the act of taking medication to alleviate symptoms, without any medical advice in the diagnosis, prescription or follow-up of the treatment. Therefore, the objective of this work is to identify the factors associated with the practice of self-medication caused by parents, the most used drugs, the reasons and to report the importance of the rational use of medications. The methodology used is based on a literature review in databases such as: the availability of medical information on the internet creates a favorable environment for the person to make a diagnosis and medicate on their own. These factors made the indiscriminate use of medications one of the main health problems in Brazil. Every medicine has side effects and, when ingested incorrectly, it can cause more harm than good to the body. Among the risks of self-medication, we can mention intoxication and the difficulty in diagnosing certain diseases due to the fact that some medications can mask important symptoms. Currently, many of those responsible for children use self-medication to relieve symptoms such as headache, cold and pain, resorting to home pharmacies, which contain analgesics, colds and anti-inflammatory drugs that may pose a risk to children. In Brazil, as in most countries, drugs are the main intoxication agent. They correspond to $27.86 \%$ of the cases registered per year, according to data from the National System of Toxic Pharmacological Information (Sinitox). Of the total number of cases of drug intoxication, children under the age of five represent $35 \%$.
\end{abstract}

Keywords: Self-medication; Inappropriate use of prescription drugs; Over-the-counter medications.

\section{Resumen}

La automedicación es el acto de tomar medicación para aliviar los síntomas, sin ningún consejo médico en el diagnóstico, prescripción o seguimiento del tratamiento. Por tanto, el objetivo de este trabajo es identificar los factores asociados a la práctica de la automedicación por parte de los padres, las drogas más utilizadas, las razones y reportar 
la importancia del uso racional de los medicamentos. La metodología empleada se basa en una revisión de la literatura en bases de datos tales como: la disponibilidad de información médica en internet crea un entorno propicio para que la persona realice un diagnóstico y se medicare por sí misma. Estos factores hicieron del uso indiscriminado de medicamentos uno de los principales problemas de salud en Brasil. Todos los medicamentos tienen efectos secundarios y, cuando se ingieren incorrectamente, pueden causar más daño que bien al cuerpo. Entre los riesgos de la automedicación, podemos mencionar la intoxicación y la dificultad para diagnosticar determinadas enfermedades debido a que algunos medicamentos pueden enmascarar síntomas importantes. Actualmente, muchos de los responsables de la infancia utilizan la automedicación para aliviar síntomas como dolor de cabeza, resfriado y dolor, recurriendo a farmacias caseras, que contienen analgésicos, resfriados y fármacos antiinflamatorios que pueden suponer un riesgo para los niños. En Brasil, como en la mayoría de los países, las drogas son el principal agente de intoxicación. Corresponden al 27,86\% de los casos registrados por año, según datos del Sistema Nacional de Información Farmacológica de Tóxicos (Sinitox). Del total de casos de intoxicación por drogas, los niños menores de cinco años representan el 35\%.

Palabras clave: Automedicación; Uso inapropiado de medicamentos recetados; Medicamentos de venta libre.

\section{Introdução}

Medicamentos são bens de saúde, seu aproveitamento pela sociedade é influenciado por vários motivos, entre eles o aumento da expectativa de vida. Devem ser compreendidos como instrumentos de promoção, recuperação e manutenção do bem-estar (Arrais et al., 2016).

A automedicação é a utilização de medicamentos por conta própria ou por indicação de pessoas não habilitadas para tratamento de doenças, com o objetivo de tratar ou aliviar sintomas mesmo de promover a saúde, independente da prescrição. A maioria desses medicamentos são administrados de maneira incorreta como, tempo e o intervalo de utilização, erros na dosagem ou ingerir com outro tipo de alimento. Nos dias de hoje os responsáveis pelas crianças utilizam a automedicação para o conforto de sintomas como dores, febre e resfriado, utilizando farmácia domiciliares as quais vendem os medicamentos livres de prescrição (MIPS). As farmacinhas caseiras geralmente armazenadas em ambientes não adequados, influenciando o uso irracional e facilitando a automedicação com riscos de toxicidade principalmente em crianças (Filho \& Júnior, 2013).

Ao optar pela automedicação, pode considerar a favor do sistema de saúde ajudando a desaglomerar os atendimentos médicos e de enfermagem na Unidades Básicas de Saúde (UBS), levando ao alivio de pequenos sintomas de indisposição do paciente, entretanto, quando não apropriado pode acarretar riscos potenciais a saúde, como alergias, intoxicações e o principal de todos, o mascaramento da doença bem como alongar o tempo para a busca do serviço de saúde em situações com maior gravidade, particularmente, quando se trata de crianças (Lima et al., 2019).

Com isso, Santos, Freitas e Eduardo (2015), automedicar-se é independente de classe social, justificada pelo fácil acesso dos fármacos em drogarias sem prescrição e pela dificuldade de atendimentos médicos no Sistema Único de Saúde (SUS).Ressaltando que o medicamento usado de forma errada torna-se um veneno para a criança, ocasionando reações adversas, sintomas esses que podem serem confundidos com sintomas de doenças, atrasar diagnósticos importantes e ainda aumentar a resistência bacteriana no caso dos antibióticos.

A automedicação em crianças é uma prática que pode levar a vários prejuízos à saúde, pois é necessário a orientação de um profissional habilitado, atenção dos responsáveis (pais e cuidadores). Uma pesquisa constatou que $71,42 \%$ das crianças foram medicadas previamente a atendimento profissional pelos pais, mostrando o elevado índice dessa prática. A faixa etária de maior prevalência é em menores de cinco anos, isso justifica a possibilidade de reaproveitamento de antigas prescrições aos irmãos mais velhos (Silva et al., 2018).

Com isso demonstrar o potencial dos riscos quanto a automedicação, justifica a elaboração desse artigo, com o objetivo de identificar os motivos e as classes farmacológicas mais frequente na prática da automedicação. 


\section{Metodologia}

Trata-se de uma revisão bibliográfica baseada nos conceitos de Marconi e Lakatos (2011); que tem como o objetivo analisar um assunto com visões críticas daquilo que está escrito. Nesse sentido, para atingir o objetivo proposto, foi definido os seguintes critérios: Identificar os fármacos mais administrados, quais fatores associados a prática da automedicação ocasionada pelos pais e relatar a importância do uso racional de medicamentos.

A identificação dos artigos será feita através de pesquisas em bancos de dados como; Pubmed (National Library of Medicine National Institutes of Health), Scielo (Biblioteca Eletrônica Scientific Eletronic Library Online), Google acadêmico. Os critérios de seleção: artigos originais fidedignos com o tema proposto, artigos em português entre o ano de 2011 a 2021. Para os critérios de exclusão: artigos não condizentes com o tema, publicações de anos anteriores, e artigos em inglês e espanhol. Os períodos da pesquisa foram de agosto a novembro de 2021.

Os dados foram examinados em três etapas:

- Primeira etapa: verificação de artigos nas bases de dados, que possuem em seu título e resumo as palavras chaves e os termos livres referentes ao assunto abordado e exclusão dos que não se enquadraram nos critérios de inclusão.

- Segunda etapa: seleção dos quais se enquadram nos critérios de inclusão para uso na pesquisa.

- Terceira etapa: leitura na totalidade dos artigos e seleção dos que apresentam o assunto apropriado para esta pesquisa.

\section{Resultados e Discussão}

Abaixo a demonstração com as principais características de artigos analisados após critérios de inclusão e exclusão, com os principais objetivos de estudo que são caracterizados por quais medicamentos mais usados na automedicação pelos pais ou responsáveis, a idade média nas crianças que isso ocorre, e por quais motivos mais frequentes e o que levam aos responsáveis a praticar a automedicação nos filhos sem a prescrição médica (Quadro $1)$.

Quadro 1. Características de artigos publicados acerca da automedicação infantil.

\begin{tabular}{|c|c|c|c|}
\hline Autor, Ano & Título & Objetivos & Principais Resultados \\
\hline Cruz et al, 2014 & $\begin{array}{l}\text { Uso de medicamentos entre } \\
\text { crianças de } 0-14 \text { anos: } \\
\text { estudo de base } \\
\text { populacional. }\end{array}$ & $\begin{array}{l}\text { Identificar a prevalência, o } \\
\text { consumo de medicamentos } \\
\text { sem prescrição, principais } \\
\text { grupos e classes } \\
\text { farmacêuticas mais usados e } \\
\text { quais fatores que induzido a } \\
\text { essa prática. }\end{array}$ & $\begin{array}{l}\text { A prevalência de consumo foi de } 56,57 \% \text { a idade média dos } \\
\text { indivíduos que consumiram medicamentos foi de dois a sete } \\
\text { anos }(38,9 \%) \text {, e de sete a quatorze anos }(43,9 \%) \text { naqueles que } \\
\text { utilizam automedicação. Os principais fatores que } \\
\text { influenciaram são: resfriado comum, gripe, tosse, congestão } \\
\text { nasal, febre, cefaleia e cólica abdominal. Os medicamentos } \\
\text { usados com maior frequência são: analgésicos, antipiréticos, } \\
\text { antibióticos sistêmicos, antialérgicos, antitérmicos, } \\
\text { descongestionantes e expectorantes. As variáveis em relação a } \\
\text { automedicação são as características sociodemográficas e } \\
\text { associado a baixa renda mensal familiar. }\end{array}$ \\
\hline $\begin{array}{c}\text { Filho \& } \\
\text { Júnior, } 2013\end{array}$ & $\begin{array}{c}\text { Automedicação em } \\
\text { crianças de zero a cinco } \\
\text { anos: Fármacos mais } \\
\text { administrados, } \\
\text { conhecimento, motivos e } \\
\text { justificativas. } \\
\end{array}$ & $\begin{array}{c}\text { Pontuar quais } \\
\text { medicamentos } \\
\text { administrados, justificativas } \\
\text { e motivos dos pais ou } \\
\text { responsáveis pela } \\
\text { automedicação. } \\
\end{array}$ & $\begin{array}{c}\text { Os medicamentos mais utilizados foram: Dipirona, paracetamol, } \\
\text { xaropes expectorantes, a justificativa colocada é para amenizar } \\
\text { os sintomas como febre, tosse, inflação na garganta, dores em } \\
\text { geral, vômito, cólica. }\end{array}$ \\
\hline $\begin{array}{c}\text { Goulart et al., } \\
2012\end{array}$ & $\begin{array}{c}\text { Automedicação em } \\
\text { menores de cinco anos em } \\
\text { municípios do Pará e Piauí: } \\
\text { Prevalência e fatores } \\
\text { associados. }\end{array}$ & $\begin{array}{l}\text { Medir a prevalência e } \\
\text { identificar os fatores } \\
\text { associados a automedicação. }\end{array}$ & $\begin{array}{l}\text { A prevalência da automedicação por parte das mães nos } \\
\text { municípios estudados ocorreu sobretudo da dificuldade de } \\
\text { realização de consulta médica. }\end{array}$ \\
\hline
\end{tabular}




\begin{tabular}{|c|c|c|c|}
\hline $\begin{array}{c}\text { Medeiros, } \\
\text { Pereira \& } \\
\text { Medeiros, 2011 }\end{array}$ & $\begin{array}{c}\text { Vigilância em saúde na } \\
\text { enfermagem: o caso das } \\
\text { medicações sem prescrição } \\
\text { em crianças. }\end{array}$ & $\begin{array}{c}\text { Descrever os motivos que } \\
\text { levaram as mães a } \\
\text { automedicar seus filhos }\end{array}$ & $\begin{array}{c}\text { Principais motivos que influenciaram na automedicação: Febre } \\
(43 \%, \text {, experiência anterior (43\%) e gripe (14\%). Experiência } \\
\text { anterior colocado como um dos fatores acima, é por ser muito } \\
\text { citado quando o tratamento correto de doenças que resultam em } \\
\text { restaurar a saúde, estimula alguns pais a passarem em frente a } \\
\text { prescrição para outros. }\end{array}$ \\
\hline $\begin{array}{c}\text { Silva et al., } \\
2016\end{array}$ & $\begin{array}{c}\text { Intoxicações } \\
\text { medicamentosas em } \\
\text { crianças de 0 a 4 anos } \\
\text { atendidas pelos centros de } \\
\text { informações } \\
\text { toxicológicas. }\end{array}$ & $\begin{array}{c}\text { Identificar agentes causadores } \\
\text { de intoxicações. }\end{array}$ & $\begin{array}{c}\text { Os medicamentos usados de forma incorreta dão os principais } \\
\text { agentes causadores de intoxicação na faixa etária citada, uma } \\
\text { vez que esse período é a fase de curiosidade que tudo levam a } \\
\text { boca. }\end{array}$ \\
\hline
\end{tabular}

Fontes: Autores (2021).

\subsection{Automedicação, dados epidemiológicos e as classes farmacêuticas mais envolvidas e os riscos}

A automedicação é o ato de ingerir medicamentos pela iniciativa do enfermo ou de seu responsável, para que possa lhe trazer benefícios no alivio de sintomas ou tratamento de doenças, dessa forma as prescrições e orientações médicas são substituídos indevidamente por sugestões de medicamentos por pessoas não autorizadas como familiares, amigos ou outros (Domingues et al., 2017).

Mesmo que a prática de se automedicar seja entendida como uma "solução" para diminuição imediato de sintomas, leva a consequências mais perigosas do que se imagina, resultando em graves danos s saúde, possui riscos inerentes Acarretando riscos ao paciente influenciando também nos custos de gastos com saúde devido as reações adversas (Andrade et al., 2020).

É comum o hábito de guardar medicamentos em domicílio, refletindo no uso irracional uma vez que um em cada quatro usuários usam medicamentos de forma errônea. Vale ressaltar sobre a importância de que o mal armazenamento de medicamentos pode refletir nas alterações físico-químicas podem sofrer por variações de exposição a luz, umidade e calor. Acultura de guardar os medicamentos na cozinha e no banheiro podem influenciar nesses riscos. Visto que a estocagem de fármacos em domicilio pode ter relação às intoxicações, inclusive acidentais, tendo cuidados criterioso em residências com a presença de crianças (Cruz et al., 2017).

No Brasil (2013), o Sistema Nacional de Informações Tóxico-Farmacológicas (Sinitox/Fiocruz) registrou, apenas em 2017, cerca de 20 mil casos de intoxicação por uso de medicamentos e 50 mortes, correspondendo a uma letalidade de $0,25 \%$. No mesmo ano, os medicamentos foram a primeira causa de intoxicação humana por agente tóxico, sendo responsável por $27,11 \%$ do total de casos registrados deste tipo de toxicose. Quanto à faixa etária, percebeu-se uma predominância de crianças menores de 4 anos e jovens adultos (20 a 29 anos) como os grupos que sofreram de envenenamento por medicamentos em 2017 (Xavier et al., 2021).

O Sistema Nacional de Informações Tóxico-Farmacológicas (SINTOX), mais de 20 crianças por dia são vítimas de intoxicações, consequência do uso inadequado de medicamentos, fato que é indicador de que seus pais não têm informações quanto a dosagem e o armazenamento adequado dos medicamentos, o crescimento de informações via internet induzem aos pais a automedicar os seus filhos, resultando assim uma intoxicação intencional ou acidental (Santana, Bochner \& Guimarães, 2011).

Em crianças, a intoxicação por medicamentos é um problema de saúde de grau perigoso e integra uma das mais rotineiras emergências toxicológicas e seu acontecimento é devido a vários fatores. Estudos mostram um elevado índice de envenenamento de forma acidental na faixa etária de 0 a 4 anos. Visto que intoxicações de forma geral acometem em todos os seres humanos, porém as medicamentosas acometem mais as crianças dessa faixa. (Silva et al., 2016).

$\mathrm{Na}$ infância os acidentes relacionados as intoxicações medicamentosas são bastante comuns. Os aumentos de informações por meio da internet influenciam alguns pais a medicarem seus filhos de maneira irrestrita. Líquidos coloridos, 
comprimidos em forma que dão aparência de doces com sabor de frutas e formato de bichinhos colaborando assim para intoxicações acidentais. Embalagens coloridas, o armazenamento incorreto, são algumas das formas que levam as crianças ingerirem medicamentos em grandes doses. (Silva \& Oliveira.,2018; Medeiros, Pereira \& Medeiros, 2011). Maniero et al. (2018), sugere a necessidade de intervenções que busquem promover o uso racional de medicamentos na população de forma geral, principalmente pelos profissionais da saúde.

A intoxicação é um efeito medicamentoso dose-dependente, variável com a predisposição individual do usuário e com as várias interações enzimáticas de indução ou inibição do metabolismo da droga. A intoxicação pode ser local (por exemplo, em pele e mucosas) ou pode ser sistêmica, dependendo das propriedades físico-químicas do tóxico, do seu mecanismo de ação e da via de exposição, o uso de uma substância tóxica no organismo que ocorre por ingestão de medicamentos em excesso ou acidentalmente provocando uma série de efeitos adversos e nocivos, afetando o equilíbrio do organismo (Cruz et al., 2014).

Cruz et al. (2014), descreve que as crianças são os principais usuários dos serviços de saúde nos países desenvolvidos e a relação de adoecimento se reflete no uso excessivo por automedicação, uma prática muito disseminada no Brasil, influenciada pela mídia e praticada sem prescrição médica. No Brasil, cerca de 80 milhões de pessoas são praticantes da automedicação, e está relacionado com o grau de instrução e informações a respeito de medicamentos assim como o acesso dos mesmos no sistema de saúde.

O cálculo da dose de medicamentos em crianças é de acordo com o peso, idade e quadro clínico, em vista disso há uma grande chance na automedicação o medicamento ser administrado de forma incorreta. Portanto, é de muita importância que os profissionais de saúde sejam pertinentes quanto a orientação sobre os medicamentos como ação preventiva (Souza et al., 2020).

Os medicamentos mais frequentes usados são os analgésicos, antitérmicos, xaropes expectorantes, antigripais, antibióticos e anti-inflamatórios não-hormonais, grande parte destes medicamentos estão classificados como MIP's (Medicamentos Isentos de Prescrição Médicas). Os três mais utilizados são: Paracetamol, Ibuprofeno e Dipirona. Os analgésicos e anti-inflamatórios são os fármacos são os que predominam na automedicação infantil e são os apontam maiores índices de intoxicação (Klein et al., 2020).

Os principais agravos que levaram a prática da automedicação foram de gripe e resfriado, dores na garganta e tosse, em pesquisa elaborada sobre a automedicação em crianças pelos pais, resultou que acontece principalmente pelos sintomas de febre, dores, cólicas e fármacos eficientes nos sintomas de doenças respiratórias. São vários os fatores que contribuem para a automedicação, como a dificuldade ou ausência de locomoção, a distância entre os serviços de saúde e o domicílio, superlotação, e a grande espera em filas (Matos et al., 2018).

Para Goulart et al. (2012), pode considerar que um dos fatores que facilita a automedicação é a impossibilidade de a criança receber atendimento médico. A dificuldade de deslocação até o atendimento de saúde, os obstáculos para o acesso aos serviços de saúde e a insatisfação dos atendimentos, são fatores que incentivam a realização da automedicação pelos responsáveis das crianças. Mesmo conhecedores dos riscos e perigos pelos responsáveis, que expões as crianças na automedicação, é necessárias ações educativas e instrutivas junto aos pais, sejam estabelecidas para estimular e fortalecer o conhecimento da população em geral e assim minimizar os riscos (Silva et al, 2011).

\section{Conclusão}

A automedicação se tornou um grave problema de saúde pública no Brasil, atingindo com mais severidade a população infantil. No caso da população infantil, a situação se agrava pelas peculiaridades do organismo infantil e também pelo alto risco de intoxicação a que as famílias brasileiras estão expostas. O uso de medicamentos sem prescrição médica apresenta uma série de perigos, independentemente da idade de quem está fazendo seu uso. 
Em crianças essa situação pode ser ainda mais perigosa, devido, entre outros fatores, à dificuldade da criança em expressar o que realmente está sentindo. Muitos medicamentos são coloridos e adocicados, o que pode atrair a atenção da criança, que pode fazer seu uso quando um responsável não estiver presente. Além disso, muitos responsáveis oferecem medicamento para crianças dizendo que se trata de balas, o que pode fazê-las pensar que se trata de algo inofensivo e que pode ser consumido sem nenhum problema. Se não utilizados em doses adequadas, muitos medicamentos podem provocar intoxicações. Além disso, se a dose for muito baixa, pode não ser suficiente para tratar um problema.

Outro ponto importante que merece destaque é a intoxicação acidental devido à presença de medicamentos nas residências. E as explicações para o agravamento da automedicação em crianças, além das intoxicações, se devem ao fato da indução de resistência bacteriana e de mascarar sintomas clínicos de doenças. A justificativa é o metabolismo acelerado das crianças, que aumenta a absorção e a ação dos princípios ativos das drogas em seu organismo. Esses riscos incluem ainda as interações com outros medicamentos já usados, efeitos adversos e intoxicações com custos para a saúde, atrasando ou dificultando o diagnóstico e a abordagem terapêutica correta.

Este estudo consiste em demonstrar a necessidade e a importância do desenvolvimento de políticas públicas voltadas para ações na Pediatria. $\mathrm{O}$ cuidado da criança começa com a orientação efetiva de seus cuidadores, o que pode ser atingido por meio de um sistema de saúde comprometido com a eficiência e a qualidade.

Em pesquisas futuras sugere-se estabelecer fatores como: socioeconômico e educacional de pais e/ou responsáveis podem impactar na automedicação infantil, ocasionando sérios problemas como intoxicações medicamentosas.

\section{Referências}

Arrais, P. S. D., Fernandes, M. E. P., Pizzol, T. da S. D., Ramos, L. R., Mengue, S. S., Luiza, V. L., Tavares, N. U. L., Farias, M. R., Oliveira, M. A. \& Bertoldi, A. D. (2016). Prevalência da automedicação no Brasil e fatores associados. Revista de Saúde Pública, 50(2), 1s-11s.

Brasil. Sistema Nacional de Informações Toxico-farmacológicas. (2013). Casos registrados de Intoxicação Humana por Agente Tóxico e Faixa Etária [Internet]. https://sinitox.icict.fiocruz.br/sites/sinitox.icict.fiocruz.br/files//Brasil7_0.pdf

Cruz, M. J. B., Azevedo, A. B., Bodevan, E. C., Araújo, L. U. \& Santos, D. F. (2017). Estoque doméstico e uso de medicamentos por crianças no Vale do Jequitinhonha, Minas Gerais, Brasil. Saúde em debate, 41(114), 836-847.

Cruz, M. J. B., Dourado, L. F. N., Bodecan, E. C., Andrade, R. A \& Santos, D. F. Uso de medicamentos entre crianças de 0-14 anos: estudo de base populacional. Jornal de Pediatria, 90(6), 608-615.

Domingues, P. H. F., Galvão. T. F., Andrade, K. R. C., Araújo, P. C., Silva, M. T. \& Pereira, M. G. (2017). Prevalência e fatores associados à automedicação em adultos no Distrito Federal: estudo transversal de base populacional. Epidemiologia e Serviços de Saúde, 26(2), 319-330.

Filho, P. C. P. T. \& Júnior, A. do C. P. (2013). Automedicação em crianças de zero a cinco anos: fármacos administrados, conhecimentos, motivos e justificativas. Escola Anna Nery, 17(2), 291-297.

Goulart, I. da C., Cesar, J.A., Gonzalez-China, D.A. \& Neumann, N.A. (2012). Automedicação em menores de cinco anos em municípios do Pará e Piauí: prevalência e fatores associados. Revista Brasileira de Saúde MaternoInfantil, 12(2), 165-172.

Klein, K., Souza, N. S., Ribeiro, A. C. \& Silva, E. B. (2020). Automedicação em crianças de zero a cinco anos: práticas de seus cuidadores/familiares. Research, Society and Development, 9(7), 1-20.

Lima, M. F. P., Lima, D. B. S., Oliveira, F. B., Oliveira, C. C., Macêdo, M. A. \& Pereira, T. K. A. (2019). A prática da automedicação em criança por pais e responsáveis. Holos, 35(5), 1-13. Doi: 10.15628/holos.2019.5120

Matos, J. F., Pena, D. A. C., Parreira, M. P., Santos, T. do C. \& Coura-Vital, W. (2018). Prevalência, perfil e fatores associados à automedicação em adolescentes e servidores de uma escola pública profissionalizante. Cadernos Saúde Coletiva, 26 (1), 76-83.

Maniero, H. K., Martins, A. A., Melo, A. C., Paz, L. P. da S., Schraiber, R. de B. \& Galato, D. (2018). Uso de medicamentos em crianças de zero a cinco anos de idade residentes no município de Tubarão, Santa Catarina. Revista Paulista de Pediatria, 36(4), 437-444.

Medeiros, R. A., Pereira, V. G. \& Medeiros, S. M. (2011). Vigilância em saúde na enfermagem: o caso das medicações sem prescrição e crianças. Escola Anna Nery, 15(2), 233-237.

Santana, R. A. L., Bochner, R., Guimarães, M. C. S. (2011). Sistema nacional de informações tóxico-farmacológicas: o desafio da padronização dos dados. Ciência \& Saúde Coletiva, 16(1), 1191-1200. 
Research, Society and Development, v. 10, n. 15, e581101523443, 2021

(CC BY 4.0) | ISSN 2525-3409 | DOI: http://dx.doi.org/10.33448/rsd-v10i15.23443

Santos, P. N. M., Freitas, R. F. \& Eduardo, A. M. de L. N. (2015). Automedicação infantil: conhecimento e motivação dos pais. Revista Multitexto, 3(1), 6572 .

Silva, J. G. da, Gomes, G. C., Costa A. R., Juliano, L. F., Aruda, C. P. \& Carvalho, L. N. (2018). A prática da automedicação em crianças por seus pais: Atuação da enfermagem. Revista de enfermagem UFPE on line, 12(6), 1570-1577.

Silva, I. M., Catrib, A. M. F., Matos, V. C. \& Gondim, A. P. S. (2011). Automedicação na adolescência:um desafio para a educação em saúde. Ciência \& Saúde Coletiva, 16(1), 1651 - 1660.

Silva, F. S. Oliveira, D. A., Augusto, M. A. \& Fernandes, E. R. L. (2016). Intoxicações medicamentosas em crianças de 0 a 4 anos atendidas pelos centros de informações toxicológicas. Catussaba - Revista Cietifica da Escola de Saúde, 5(1), 121-132.

Silva, T. J. \& Oliveira, V. B. (2018). Intoxicação medicamentosa infantil no Paraná. Visão Acadêmica, 19(1), 51-61.

Souza, A. L. D. M., Batista, F. F. de A., Tacla, M. T. G. M., Cardelli, A. A. M. \& Ferrari, R. A. P. (2020). Medication use in children below one-year-old. Revista Sociedade Brasileira de Enfermeiros Pediatras, 20(1), 31-39.

Xavier, M. S., Castro, H. N., Souza, L. G. D., Oliveira, Y. L., Tafuri, N. F. \& Amâncio, N. de F. G. (2021). Automedicação e o risco à saúde: uma revisão de literatura. Brazilian Journal of Health Review, 4(1), 225-240. 